\title{
An Algorithm for Detecting Collision Risk between Trucks and Pedestrians in the Connected Environment
}

\author{
Seung-oh Son $\mathbb{D},{ }^{1}$ Juneyoung Park $\mathbb{D},,^{1,2}$ Cheol Oh $\mathbb{D},{ }^{1,2}$ and Chunho Yeom $\mathbb{D}^{3}$ \\ ${ }^{1}$ Department of Smart City Engineering, Hanyang University, 15588 Ansan, Republic of Korea \\ ${ }^{2}$ Department of Transportation and Logistics Engineering, Hanyang University, 15588 Ansan, Republic of Korea \\ ${ }^{3}$ International School of Urban Sciences, University of Seoul, 02592 Seoul, Republic of Korea \\ Correspondence should be addressed to Juneyoung Park; juneyoung@hanyang.ac.kr
}

Received 25 August 2021; Accepted 16 October 2021; Published 28 October 2021

Academic Editor: Xiaoyue Liu

Copyright (c) 2021 Seung-oh Son et al. This is an open access article distributed under the Creative Commons Attribution License, which permits unrestricted use, distribution, and reproduction in any medium, provided the original work is properly cited.

\begin{abstract}
This study develops an algorithm to detect the risk of collision between trucks (i.e., yard tractors) and pedestrians (i.e., workers) in the connected environment of the port. The algorithm consists of linear regression-based movable coordinate predictions and vertical distance and angle judgments considering the moving characteristics of objects. Time-to-collision for port workers (TTCP) is developed to reflect the characteristics of the port using the predictive coordinates. This study assumes the connected environment in which yard tractors and workers can share coordinates of each object in real time using the Internet of Things (IoT) network. By utilizing microtraffic simulations, a port network is implemented, and the algorithm is verified using data from simulated workers and yard trucks in the connected environment. The risk detection algorithm is validated using confusion matrix. Validation results show that the true-positive rate (TPR) is $61.5 \sim 98.0 \%$, the false-positive rate (FPR) is $79.6 \sim 85.9 \%$, and the accuracy is $72.2 \sim 88.8 \%$. This result implies that the metric scores improve as the data collection cycle increases. This is expected to be useful for sustainable transportation industry sites, particularly IoT-based safety management plans, designed to ensure the safety of pedestrians from crash risk by heavy vehicles (such as yard tractors).
\end{abstract}

\section{Introduction}

Technologies, educational programs, and policies designed to prevent industrial accidents have been widely implemented, and accidents are on the decline in various industrial sectors. However, accidents are still common at ports. According to the Korea Maritime Institute, the accident rate for port workers stood at 9.46 per thousand as of 2017, double the average of 4.84 for all Korean industries. This is 1.5 times greater than the rate in the transportation sector, in which traffic accidents occur more frequently. Port activities involve manual tasks associated with binding and, depending on the type of cargo, loading and unloading, and storage methods. Collisions, falling objects, and falling accidents occur frequently in ports [1]. The severity of an accident is higher compared to those in the industrial sector in general because heavy equipment is involved. The most frequent and accidental accident is collision. At a port, most heavy equipment emits a warning sound to prevent collisions in advance. This makes it difficult for the operator to recognize access equipment (such as yard tractors), and accidents in these circumstances can be fatal. In the yard area of a port, there are two main cases of collision that can occur between workers and trucks (Table 1).

Collisions between workers and equipment in port yards are similar to pedestrian-vehicle crashes on roads, which constitute a leading concern in traffic safety. Multiple algorithm-based technologies have been developed to avoid collisions, including the forward crash warning system [2-5]. The concept of time-to-collision (TTC), which is used frequently in these algorithms, is defined in relation to the preceding and following vehicles [6]. The following equation $[7,8]$ provides a mathematical description of the concept:

$$
\operatorname{TTC}=\frac{h-L}{V_{F}-V_{P}},
$$


TABLE 1: Examples of collision-risk situations between pedestrian and truck.

No. Situation
$\begin{aligned} & \text { The worker is working or waiting in the yard. The yard tractor is driving on the road } \\ & \text { at normal speed and approaching the worker's location. The worker is not aware of } \\ & \text { the approaching tractor. }\end{aligned}$
$\begin{aligned} & \text { The worker is on the move. The yard tractor is driving on the road at normal speed } \\ & \text { and approaching the worker's location. The worker is not aware of the approaching } \\ & \text { tractor. }\end{aligned}$

where $h$ is the space headway and $L$ is the length of the preceding vehicle. $V_{F}$ and $V_{P}$ refer to the speeds of the following and preceding vehicles, respectively. The TTC assumes that the preceding vehicle maintains a constant speed. As a result, traditional TTCs are limited in their ability of expressing dynamic situations in which speed changes rapidly on real roads. To address this shortcoming, researchers have developed complementary algorithms such as inverse TTC, time-exposed TTC, and time-integrated TTC, for use in collision-risk detection technologies [9-13].

TTC is used not only in detection algorithms as an indicator of real-time collisions, but also in assessing safety levels based on trajectory [14]. TTC is a representative indicator for the assessment of safety, and indicators such as postencroachment time (PET) and the deceleration rate to avoid a crash are used for analysis $[15,16]$. In 2003, Yang et al. developed a hydraulically based system for use in a rear-end collision warning and avoidance [17]. In the carfollowing situation, the warning system was designed with a condition-space-based approach. In 2018, Wu et al. developed an algorithm to determine the risk of a rear-end collision using real-time data. Unlike previous research, the $\mathrm{Wu}$ et al. study developed collision-prevention algorithms by processing real-time data that can be used in connected vehicle environments [18]. In 2019, Wu et al. used PET as an indicator for a safety analysis of the conflicting section between the bicycle road and the vehicle driveway. The authors analyzed the mechanical behavior between bicycles and vehicles to develop an algorithm that estimated crash risks and triggered a warning alarm [19]. In 2006, Oh et al. proposed a method of determining the risk of rear-end crashes using loop-detector data and applying the concept of a safe stopping distance. The relationship between the preceding and following vehicles in a collision can be expressed mathematically [20]. In 2016, Lee and Yeo developed a collision-warning algorithm based on a multilayer perceptron neural network. An algorithm was proposed to provide collision-warning information by predicting the right of influence and deceleration of passage time and utilizing segment information collected by a roadside communication unit [7]. In 2020, Yue et al. conducted a study from the vehicle's perspective using a pedestrian-tovehicle (P2V) driving simulator to predict and avoid pedestrians in pedestrian-vehicle collisions. Research has been conducted to improve $\mathrm{P} 2 \mathrm{~V}$ technology from the driver's perspective, which differs from the pedestrian's perspective, to increase pedestrian safety [21]. In 2019, Wu et al. proposed a system of risk assessment for pedestrian-vehicle collisions. Using data collected from Lidar and other sensors, they predicted pedestrian crossing intentions using a trajectory prediction model and analyzed risks based on a dynamic Bayesian network [22].

Other studies developed vehicle-to-vehicle communications systems that assume a smooth communications environment and perform a rear-end collision-risk analysis based on intervehicle information and collected communications [23-25]. Sensor-based studies that prioritize nearfield networks to determine accident risks are already being used throughout the industry. However, research on longdistance communication using global positioning satellite (GPS) data is still being carried out with an eye to commercialization as it requires technical improvements, such as less battery maintenance and superior GPS reception. In 2017, Chen et al. calculated alternative safety indicators, such as TTC and PET between vehicles and pedestrians, to assess safety at intersections under specific conditions and times [26]. The study featured an analysis of traditional TTC problems and $\mathrm{P} 2 \mathrm{~V}$ relationships by applying formulas. In 2019, Li et al. attempted to predict the maneuverability of adjacent vehicles through inferences based on probability methodologies such as Bayesian networks. The resulting dynamic features have been presented and are considered significant advancements in terms of real-time risk management [27].

Most TTC-based algorithm technologies have been approached from a vehicle perspective $[7,17,21,23$, $26,28,29]$ and little relevant research has been conducted to help pedestrians avoid collisions with vehicles $[26,30]$. In 
this study, a vehicle-collision avoidance algorithm is proposed from the perspective of the pedestrian (worker). In a port, devices can be distributed to workers to facilitate the collection and management of data. The device is developed using IoT-based GPS technology and can determine the realtime location of workers and equipment. These IoT-based collision-risk detection technologies are expected to be applied first in traffic environments that guarantee the safety of pedestrians. The algorithm technology described in this study is expected to play an important role in planning pedestrian traffic safety measures in future sustainable pedestrian-oriented traffic environments.

The purpose of this study is to develop detection techniques that can predict pedestrian collision risks using connected technology under special conditions, such as lowtraffic ports or nonsignal intersections. The movable objects of the port terminal travel in a designated direction through a designated road, but pedestrians cannot specify the direction of progress due to their characteristics. Considering this, in this study, the speed, direction, and location of the equipment are defined by the change in relative distance as predictions. A collision-risk detection algorithm is developed reflecting the defined concept, which is validated using microtraffic simulations. With reference to the performance of the device that can be applied to the field, a simulation scenario with different data collection cycles is designed. Validation of the algorithm was performed by considering differences in risk detection accuracy on a collection cycle basis.

\section{Methodologies}

2.1. Prediction of Relative Space. In this study, risk indicators were calculated based on traditional TTC concepts. Traditional TTCs are used not only for crash risk detection, but also for conflict-based safety analysis at intersections and certain other sections. This study used a risk detection methodology using latitude and longitude coordinates. The relative distance is then calculated to determine the risk as in the following equation. Euclidean distances were used to calculate the distance between coordinates:

$$
l_{r}=\sqrt{\left(x_{p}-x_{e}\right)^{2}+\left(y_{p}-y_{e}\right)^{2}},
$$

where $l_{r}$ is the distance between port worker and equipment and $\left(x_{p}, y_{p}\right)$ and $\left(x_{e}, y_{e}\right)$ are the coordinates of the port worker and equipment (based on geographical distances), respectively. This study produced a changing relative distance-based TTC between worker and equipment. Alternative safety indicators were then proposed to respond to and avoid expected collisions. Considering the spatial characteristics of the port, a general straight section predicted future coordinates using a linear regression model. In many previous studies of collision-warning algorithms, the perception reaction time (PRT) is assumed to be between 0 and $2.5 \mathrm{~s}$ [31-33]. In this study, response time is set in consideration of the PRT by taking into account the vehicles, the work of the port workers, and mobility characteristics. The movement of the equipment is predicted for more than $10 \mathrm{~s}$ from detection and recognition of, and response to, collision risk, and verification of the research methodology is carried out.

A criterion of $10 \mathrm{~s}$ for detecting accident risks is set given the characteristics of the port container yards. A one-yard block is compartmentalized so that it can accommodate 15 containers (20 ft) horizontally and six vertically. A block is $90 \mathrm{~m}$ wide and $15 \mathrm{~m}$ long. The speed limit of moving equipment (such as yard tractors) in the port is $30 \mathrm{~km} / \mathrm{h}$ $(8.3 \mathrm{~m} / \mathrm{s})$. The maximum distance the equipment can travel in $10 \mathrm{~s}$ is therefore $83 \mathrm{~m}$. Because the width of a one-yard block is $90 \mathrm{~m}$, the time of $10 \mathrm{~s}$ in the algorithm is the maximum prediction time to ensure that all movement up to the next direction selection can be predicted. Because the port is a difficult working environment to recognize approaching equipment, collision accidents caused by low speeds continue to occur. For example, if a yard tractor moves at a speed of $5 \mathrm{~km} / \mathrm{h}$, it can travel $13.9 \mathrm{~m}$ in $10 \mathrm{~s}$. Due to the reduction of the hazard radius and sudden acceleration and deceleration behavior, it is necessary to select the forecast range for the next $10 \mathrm{~s}$. In this study, a complementary TTC is proposed based on predicting a minimum collision distance with workers within $10 \mathrm{~s}$. The minimum relative distance based on recursive algorithms can be found in the following equation:

$$
l_{r, t}=\sqrt{\left(x_{p, t}-x_{e, t}\right)^{2}+\left(y_{p, t}-y_{e, t}\right)^{2}}, \quad(0<t<10 s),
$$

where $l_{r, t}$ is the relative distance between worker and equipment at any time $t$ and $\left(x_{p, t}, y_{p, t}\right)$ and $\left(x_{e, t}, y_{e, t}\right)$ are the coordinates of port worker and equipment, respectively, at any time $t$. The collision risk is calculated based on the minimum value among the relative distances calculated by location information within $10 \mathrm{~s}$ of the future.

In order to use the collected position coordinates for analysis, a transformation work is required. Because the latitude and longitude coordinates used in this study are spherical coordinates, they should be converted to Cartesian coordinates to obtain relative distances. The haversine formula is used to convert orthogonal coordinates to the Cartesian system, and the relative distance is calculated after conversion. In this study, a regression model with recursive concept is developed to predict location coordinates. In other words, it is a method of using historical location information to produce future forecast information. In Figure 1 , the recursive concept is illustrated.

When the current time is $t_{0}$ and the historical coordinates recorded before $t$ seconds are supplied, a linear regression model can be constructed with the slope dependent on the amount of change and the regression coefficient and dependent variables as the predicted coordinates. The $y$-axis coordinates (latitude) and $x$-axis coordinates (longitude) were predicted by building separate equations as follows:

$$
\begin{aligned}
y_{\text {predicted }, e, t} & =\frac{\left(y_{e, t}-y_{e, t+1}\right)}{\left(x_{e, t}-x_{e, t+1}\right)} \cdot x_{\text {predicted }, e, t}+e \\
& =f^{\prime}(t) \cdot x_{\text {predicted }, e, t}+e,
\end{aligned}
$$




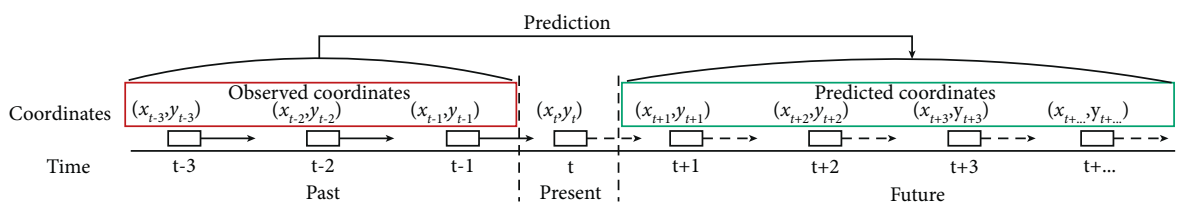

Figure 1: A recursive algorithm for predicting coordinates by time.

where $y_{\text {predicted,e,t }}$ and $x_{\text {predicted,e,t }}$ are the predicted $y$-axis (latitude) and $x$-axis (longitude) coordinates of equipment, respectively. $e$ is a residual, and $f^{\prime}(t)$ is the regression coefficient of the prediction model, such that

$$
\begin{aligned}
d_{t} & =\frac{\left|a x_{\text {predicted }}+b y_{\text {predicted }}+c\right|}{\sqrt{a^{2}+b^{2}}} \\
& =\frac{\left|f^{\prime}(t) \cdot x_{\text {predicted }, t}-y_{\text {predicted }, t}+e\right|}{\sqrt{f^{\prime}(t)^{2}+(-1)^{2}}}, \\
x & =\frac{-b \pm \sqrt{b^{2}-4 a c}}{2 a} \theta_{t}=a \sin \left(\frac{d_{t}}{l_{r}}\right) \times \frac{180^{\circ}}{\pi},
\end{aligned}
$$

where $d_{t}$ is the vertical distance between the predicted directional line of the moving equipment and the worker. $\theta_{t}$ is the degree between the equipment and the worker (Figure 2).

2.2. Time-to-Collision for Port Workers (TTCP). The relative distance $\left(l_{r}\right)$ from the current time to the predicted coordinates after $10 \mathrm{~s}$ has been aggregated to define the time at which $l_{r}$ is minimal, as seen in equation (7). In addition, changes in $l_{r}$ and relative velocity $\left(v_{r}\right)$ between the forecast coordinates for a worker and equipment can provide a realtime rate of change $\left(v_{l, \Delta t}\right)$ of the current relative speed. The time-to-collision for port workers (TTCP) can be calculated using the relationship between relative distance and realtime rate of change:

$$
\mathrm{TTCP}=t_{\min }-t_{0}=\frac{l_{r, t}}{\left(l_{r, t_{2}}-l_{r, t_{1}}\right) /\left(t_{2}-t_{1}\right)}=\frac{l_{r, t}}{v_{r, \Delta t}},
$$

where $t_{\min }$ is the time at which the relative distance between objects for the future $10 \mathrm{~s}$ is minimum. $t_{0}$ is current time. $l_{r, t}$ is relative distance, and $v_{r, \Delta t}$ is the relative distance reduction rate at time $t$. TTCP is the time calculated for the rate at which the relative distance and relative velocity can be obtained through the prediction coordinates and the relative velocity is reduced by the amount of change in this indicator. It is the time taken to minimize $l_{r, t}$ from the worker, assuming that the worker's presence is recognized within a certain relative distance and that the worker moves in the predicted direction at the current speed for $10 \mathrm{~s}$. The set $10 \mathrm{~s}$ is the maximum value that can detect all accessible objects. In this study, the TTCP threshold to determine the presence or absence of a dangerous situation is set to $4 \mathrm{~s}$. According to Yue et al., given a brake reaction rate of 1.25 to $1.5 \mathrm{~s}$ (from the previous study), a TTC of $2 \mathrm{~s}$ is considered a serious precrash accident [21]. In addition, given a brake response speed and the worker's response speed of $2.5 \mathrm{~s}$, a TTC of $4 \mathrm{~s}$ is

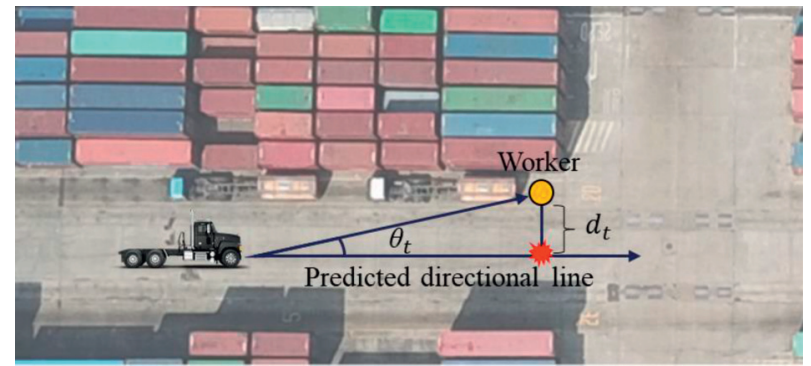

FIGURE 2: Illustration of the distance between the tractor's predicted directional line and a worker.

considered dangerous. Therefore, in this study, when the proposed TTCP is $4 \mathrm{~s}$, the situation is considered dangerous (precrash situation). Because the maximum travel distance for $4 \mathrm{~s}$ at a design speed of $30 \mathrm{~km} / \mathrm{h}$ is approximately $33.3 \mathrm{~m}$, this is almost consistent with the relative distance-based risk assessment standard of $30 \mathrm{~m}$.

\subsection{Development of a Crash Risk Detection Algorithm for Port} Workers. To detect accident risks in real time by combining the proposed alternative safety indicators such as TTCP requires defining and classifying various behaviors of workers and equipment. In this study, given the basic characteristics of accidents occurring within the port, directly relevant variables were derived and considered in the algorithm. Factors judged important and reflected in the development of algorithms are work status, location (place), distance from objects (relative distance, $l_{r}$ ), vertical distance $\left(d_{t}\right)$, degree $\left(\theta_{t}\right.$, and approach direction $\left(v_{r}\right.$.

According to Layton and Dixon (2012), the stopping sight distance is $31.2 \mathrm{~m}$ at a design speed of $30 \mathrm{~km} / \mathrm{h}$, and the typical emergency stopping distance is $14.2 \mathrm{~m}$ on dry road surfaces (with a response time of $2.5 \mathrm{~s}$ ) [31]. By referring to this standard, yard trucks within $30 \mathrm{~m}$ of the worker and approaching the worker's direction were detected as dangerous objects. In this study, an algorithm was developed by largely dividing it into two stages based on these criteria. The first is the "risk situation judgement algorithm" that detects objects approaching within a relative distance of $15 \mathrm{~m}$. The second is "collision-risk detection algorithm," which determines the designated TTCP criterion according to vertical distance $\left(d_{t}\right)$ and angle $\left(\theta_{t}\right.$. Collision-risk detection algorithm is performed only for objects determined to be dangerous by risk situation judgement algorithm. The developed algorithm is shown in Figure 3.

Figure 4 provides a diagram of the algorithm to determine the risk of collision at a port. It is the process of calculating the indicators according to changes in the 


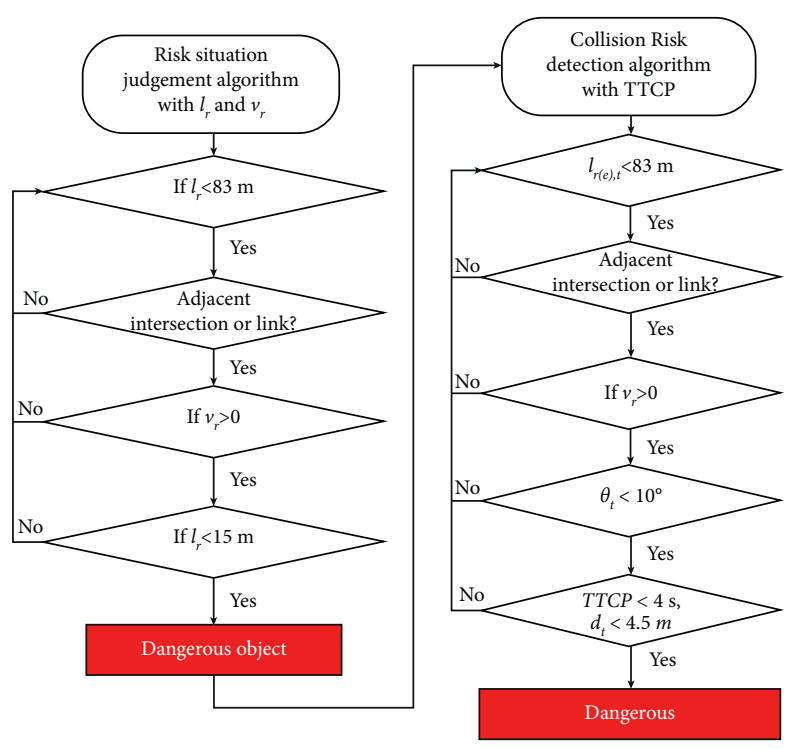

FIGURE 3: Risk detection algorithm with TTCP.
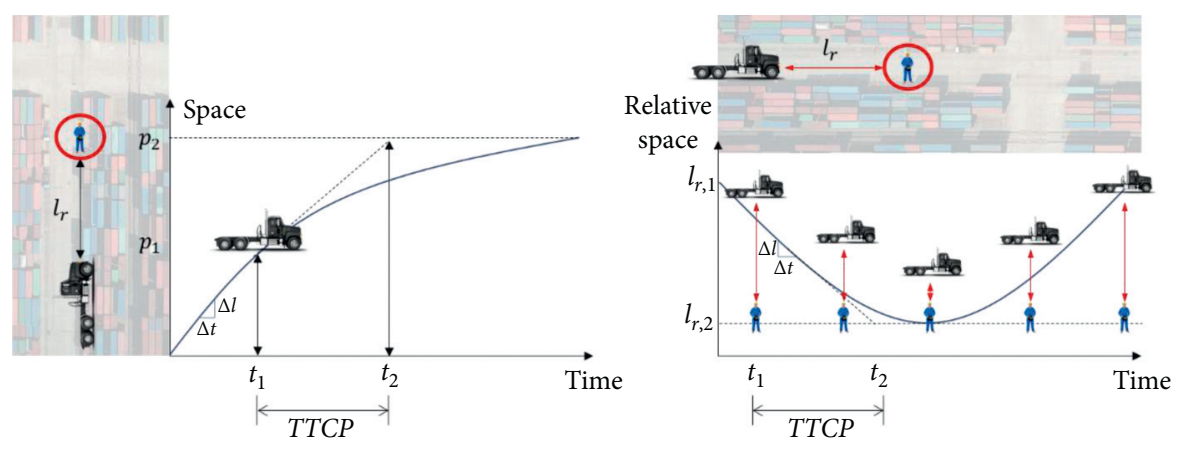

FIGURE 4: Risk detection algorithm with TTCP.

relative space between trucks and workers. This figure expresses a simple situation for understanding. In practice, the change in speed of the object can be reflected to detect changes in the TTC according to the speed.

2.4. Data Description. This study assumes a situation in which the real-time locations of workers and equipment are collected using IoT devices. This allowed us to derive the risk of a collision accident between workers and equipment in a port yard in the form of a TTC-based risk index and detection using the collected data. Technical research is currently being carried out at the port in anticipation of the introduction of IoT devices. To implement and evaluate accident-risk detection technologies in an IoT communication environment, a virtual environment is established using microtraffic simulations. Previous studies used data collected from actual roads [12, 34], where it is difficult to ensure a fully controlled environment for experimentation and implement the desired scenario. Thus, in this study, simulation analyses were performed that enabled the full implementation of the planned risk scenario. In this study, the risk of a collision within a port is divided into scenarios.
The actual environment and working characteristics were classified and analyzed to capture all possible situations between workers and equipment in the simulation network, and the risk of collision at the port is separated into four distinct scenarios. Trace data on workers and equipment were collected for each scenario using VISSIM, a microtraffic simulation program. The simulation time is set to a total length of 1,200 s for the collection of data, and analysis is performed by dividing the collection cycle into three categories, considering the communication performance of an actual IoT device.

The period for the analysis is set to $1 \mathrm{~Hz}, 2 \mathrm{~Hz}$, or $10 \mathrm{~Hz}$. To simulate the movement of equipment and yard tractors in the port, movement at the actual port is analyzed and the average speed is below $30 \mathrm{~km} / \mathrm{h}$. Network settlement and verification efforts were not carried out because the objective is to detect the risk of collision between workers and equipment in certain situations without considering intervehicle interactions and delays in traffic. The spatial background applied in this study is Busan Sinseondae Pier (Figure 5). The same spatial network is established using Google and Kakao maps, and photos are taken at realworld sites. 


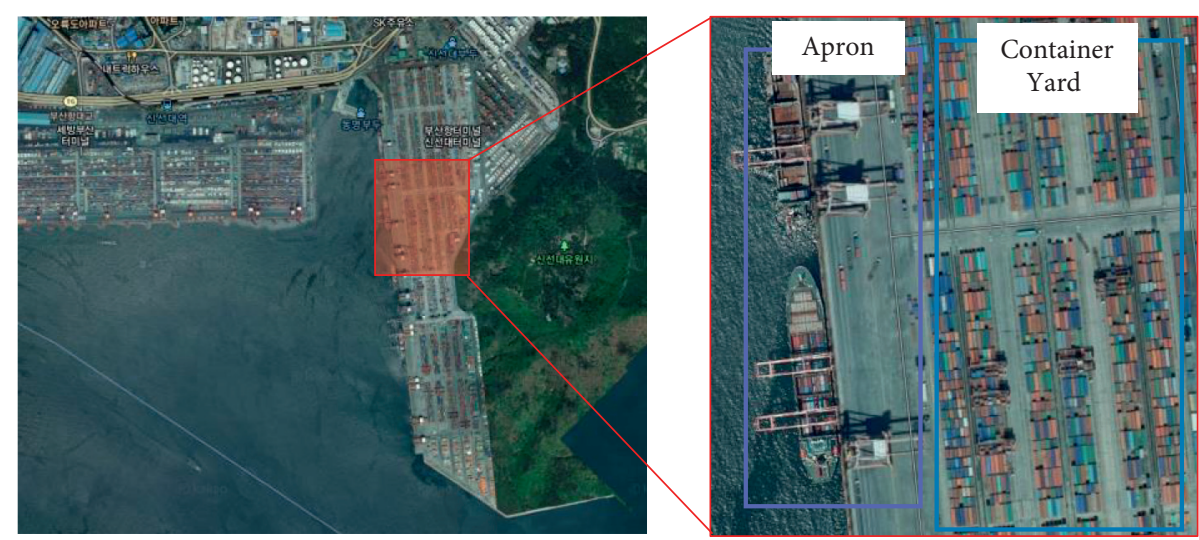

FIgure 5: Study area (Sinseondae Pier, Busan Port Terminal).

TABle 2: Confusion matrix.

\begin{tabular}{lccr}
\hline & Classification & & Actual class \\
& & Dangerous & Normal \\
\hline \multirow{2}{*}{ Prediction } & Dangerous & True positive (TP) & False positive (FP) \\
& Normal & False negative (FN) & True negative (TN) \\
\hline
\end{tabular}

\section{Results and Discussion}

Algorithm verification is carried out to detect the risk of a collision between a worker and equipment within the port using trajectory data collected in the analysis network and VISSIM. The analysis is performed by dividing the data into three scenarios according to the frequency of the information collection. Simulation experiments were conducted according to the information collection cycle. To evaluate the risk detection performance of the algorithm, the risk situation is defined as follows:

(1) If the relative distance is within $30 \mathrm{~m}$ and the relative distance is decreasing, the relative velocity $\left(v_{r}\right)$ is positive and the mobile device is approaching

(2) Relative distance within $15 \mathrm{~m}$

In this study, the confusion matrix is used to evaluate the detection performance of the algorithm. As a criterion for determining the accuracy of the predicted value, the above two situations were set as the actual class (Table 2). The confusion matrix is used to compare the number of true and false predicted values and actual values. The simulation results of algorithm are classified into four categories to evaluate detection accuracy as follows:

True positive (TP): the algorithm predicted that a situation is dangerous, and it is true

False positive (FP): the algorithm predicted that a situation is dangerous, and it is false

False negative (FN): the algorithm predicted that a situation is normal, and it is false

True negative (TN): the algorithm predicted that a situation is normal, and it is true
True positive rate $(\mathrm{TPR})=$ recall $($ sensitivity $)$

$$
=\frac{\mathrm{TP}}{\mathrm{TP}+\mathrm{FN}}
$$

False positive rate $(\mathrm{FPR})=1-$ specificity

$$
\begin{gathered}
=\frac{\mathrm{FP}}{\mathrm{FP}+\mathrm{TN}}, \\
\text { accuracy }=\frac{\mathrm{TP}+\mathrm{TN}}{\mathrm{TP}+\mathrm{FN}+\mathrm{FP}+\mathrm{TN}} .
\end{gathered}
$$

True-positive rate (TPR), also called sensitivity, refers to how accurately the algorithm classifies dangerous situations $(\mathrm{TP}+\mathrm{FN})$, as seen in equation (8). False-positive rate (FPR) indicates how accurately the algorithm classifies normal situations ( $\mathrm{TN}+\mathrm{FP})$, as seen in equation (9). Accuracy is an indicator that considers both TP and TN, which can most intuitively represent the performance of the algorithm [35], as seen in equation (10).

The numbers of samples according to the data collection cycle were 12,811 (1 Hz), 25,147 (2 Hz), and 137,254 (10 Hz), respectively. The confusion matrix and metric scores are presented in Tables 3-6. The metric scores of $1 \mathrm{~Hz}$ show $61.5 \%, 79.6 \%$, and $72.2 \%$. This score is the smallest of three data collection cycle scenarios. Compared to other cycles, $1 \mathrm{~Hz}$ has a longer prediction unit. Therefore, this result is seen as an increase in error within the prediction unit. For $2 \mathrm{~Hz}$, the data were every $0.5 \mathrm{~s}$; the metric score shows $91.8 \%$, $85.9 \%$, and $87.5 \%$. Finally, for $10 \mathrm{~Hz}$ and the shortest collection cycle, the metric score shows $98.0 \%, 85.4 \%$, and $88.8 \%$. It is obvious that the score of $10 \mathrm{~Hz}$ has the greatest accuracy among the cycles, which suggests that the shorter 
TABLE 3: Results (data acquisition cycle $=1 \mathrm{~Hz}$ ).

\begin{tabular}{lccc}
\hline \multirow{2}{*}{ Classification } & \multicolumn{2}{c}{ Actual class } \\
& & Dangerous & Normal \\
\hline \multirow{2}{*}{ Prediction } & Dangerous & 3,220 & 1,545 \\
& Normal & 2,013 & 6,032 \\
\hline
\end{tabular}

True-positive rate $(\mathrm{TPR})=0.615 ;$ false-positive rate $(\mathrm{FPR})=0.796$; accuracy $=0.722$.

TABle 4: Results (data acquisition cycle $=2 \mathrm{~Hz}$ ).

\begin{tabular}{cccc}
\hline \multirow{2}{*}{ Classification } & \multicolumn{2}{c}{ Actual class } \\
& & Dangerous & Normal \\
\hline \multirow{2}{*}{ Prediction } & Dangerous & 6,249 & 2,588 \\
& Normal & 561 & 15,748 \\
\hline
\end{tabular}

True-positive rate $(\mathrm{TPR})=0.918 ;$ false-positive rate $(\mathrm{FPR})=0.859$; accuracy $=0.875$.

TABle 5: Results (data acquisition cycle $=10 \mathrm{~Hz}$ ).

\begin{tabular}{lccc}
\hline \multicolumn{2}{c}{ Classification } & \multicolumn{2}{c}{ Actual class } \\
& & Dangerous & Normal \\
\hline \multirow{2}{*}{ Prediction } & Dangerous & 35,967 & 14,661 \\
& Normal & 724 & 85,901 \\
\hline
\end{tabular}

True-positive rate $(\mathrm{TPR})=0.980 ;$ false-positive rate $(\mathrm{FPR})=0.854$; accuracy $=0.888$.

TABLe 6: Metric scores for algorithm (data acquisition cycle $=1,2$, $10 \mathrm{~Hz}$ ).

\begin{tabular}{lccc}
\hline $\begin{array}{l}\text { Data acquisition cycle } \\
(\mathrm{Hz})\end{array}$ & $\begin{array}{c}\text { True-positive } \\
\text { rate }\end{array}$ & $\begin{array}{c}\text { False-positive } \\
\text { rate }\end{array}$ & Accuracy \\
\hline 1 & 0.615 & 0.796 & 0.722 \\
2 & 0.918 & 0.859 & 0.875 \\
10 & 0.980 & 0.854 & 0.888 \\
\hline
\end{tabular}

the collection cycle, the better the performance of the algorithm.

In summary, sensitivity analysis and comparative studies have been conducted to verify the performance of the algorithm. Sensitivity analysis shows the performance of algorithms associated with data collection cycles, which means that shorter collection cycles are better in terms of detection accuracy of algorithms. Considering current technical level such as battery and equipment size, it is difficult to supply equipment with $10 \mathrm{~Hz}$. Therefore, it is necessary to establish equipment and servers that can provide an appropriate data collection cycle in consideration of the realistic conditions of the industrial site.

\section{Conclusions}

This study developed and verified an algorithm to detect the risk of collision accidents between port workers and equipment. A risk indicator represented by TTC is selected to provide detection of the risk of collision due to equipment in situations specified by various conditions in the port.
Traditional TTCs are the simplest collision-detection replacement safety indicators and only detect hazardous situations if certain conditions are met. Various studies have suggested improving TTCs to compensate for these problems. The purpose of this study is to develop an algorithm that considers the characteristics of pedestrian behavior by calculating the TTC between workers and equipment, not TTCs, in the situation of the next lane.

As a result, the algorithm shows 61.5-98.0\% TPR, 79.6-85.9\% FPR, and $72.2-88.8 \%$ Accuracy depending on the collection cycle. The algorithm validation results indicate that the metric scores increase as the collection cycle is shortened. This means that the better the performance of individual IoT devices available in the field is, the better the risk detection level of the algorithm can be. Based on these analysis results, this study argues for the need to introduce smart port equipment following technical improvements. Because it is an algorithm developed considering the spatial characteristics of ports, it is significant in terms of safety improvement in industrial sites.

However, some limitations exist in this study. First, since it is a simulation-based algorithm study assuming conditions in the field, further verification using real-world field data is required. Second, improvements are needed on the threshold baseline setting of stopping sight distance and TTC. In this study, the criteria for studies conducted in general traffic flow environments were utilized. Therefore, the stopping sight distance and TTC settings that are optimized for a port environment are needed [30,36]. Third, the accuracy improvement of the algorithm can be performed by utilizing various prediction and verification methodologies. In this study, predictive method is used for real-time contextual judgement and algorithm optimization. However, since machine learning-based positional coordinate prediction studies have been conducted, various prediction methodologies need to be reviewed to improve the accuracy of algorithms [37]. In addition, studies of collisionprevention methodologies using similar techniques can be applied to improvements [38-40]. Future research will be considered verifying algorithms using device data collected in the field and improving services after introduction. It is expected to help prevent accidents between equipment and pedestrians at ports and other industrial sites.

\section{Data Availability}

Some or all data, models, or code that support the findings of this study are available from the corresponding author upon reasonable request.

\section{Disclosure}

This research was a part of the project titled "Development of Port Risk Prediction and Intelligent Port Safety Management Technologies".

\section{Conflicts of Interest}

The authors declare no conflicts of interest. 


\section{Acknowledgments}

This paper was funded by the Ministry of Oceans and Fisheries, Korea (20190399-08).

\section{References}

[1] J. Zhang, Â. P. Teixeira, C. Guedes Soares, and X. Yan, "Quantitative assessment of collision risk influence factors in the Tianjin port," Safety Science, vol. 110, pp. 363-371, 2018.

[2] Y. Liu, W. Wang, X. Hua, and S. Wang, "Safety analysis of a modified cooperative adaptive cruise control algorithm accounting for communication delay," Sustainability, vol. 12, no. 18 , p. 7568, 2020.

[3] T. Nakazawa, S. Tang, and S. Obana, "CCN-based inter-vehicle communication for efficient collection of road and traffic information," Electronics, vol. 9, no. 1, p. 112, 2020.

[4] L. Wang, H. Zhong, W. Ma, M. Abdel-Aty, and J. Park, "How many crashes can connected vehicle and automated vehicle technologies prevent: a meta-analysis," Accident Analysis \& Prevention, vol. 136, Article ID 105299, 2020.

[5] L. Yue, M. Abdel-Aty, Y. Wu, O. Zheng, and J. Yuan, "Indepth approach for identifying crash causation patterns and its implications for pedestrian crash prevention," Journal of Safety Research, vol. 73, pp. 119-132, 2020.

[6] R. van der Horst and J. Hogema, "Time-to-collision and collision avoidance systems," in Proceedings of the 6th ICTCT Workshop Salzburgna, Salzburg, Germany, 1994.

[7] D. Lee and H. Yeo, "Real-time rear-end collision-warning system using a multilayer perceptron neural network," IEEE Transactions on Intelligent Transportation Systems, vol. 17, no. 11, pp. 3087-3097, 2016.

[8] R. Utriainen, "The potential impacts of automated vehicles on pedestrian safety in a four-season country," Journal of Intelligent Transportation Systems, vol. 25, no. 2, pp. 188-196, 2020.

[9] H. C. Chin, S. T. Quek, and R. L. Cheu, "Traffic conflicts in expressway merging," Journal of Transportation Engineering, vol. 117, no. 6, pp. 633-643, 1991.

[10] H.-C. Chin and S.-T. Quek, "Measurement of traffic conflicts," Safety Science, vol. 26, no. 3, pp. 169-185, 1997.

[11] H. Yang, K. Ozbay, and B. Bartin, "Application of simulationbased traffic conflict analysis for highway safety evaluation," in Proceedings of the 12th WCTR, vol. 4, Lisbon, Portugal, 2010.

[12] Z. Wei, S. Xiang, D. Xuan, and L. Xu, "An adaptive vehicle rear-end collision warning algorithm based on neural network, communications in computer and information science," in International Conference on Information and Management Engineering, pp. 305-314, Springer, Berlin, Germany, 2011.

[13] R. J. Kiefer, D. J. LeBlanc, and C. A. Flannagan, "Developing an inverse time-to-collision crash alert timing approach based on drivers' last-second braking and steering judgments," Accident Analysis \& Prevention, vol. 37, no. 2, pp. 295-303, 2005.

[14] J. Hou, G. F. List, and X. Guo, "New algorithms for computing the time-to-collision in freeway traffic simulation models," Computational Intelligence and Neuroscience, vol. 2014, Article ID 761047, 8 pages, 2014.

[15] B. L. Allen, B. T. Shin, and P. J. Cooper, Analysis of Traffic Conflicts and Collisions, McMaster University, Hamilton, Canada, 1978.
[16] A. Razmpa, An Assessment of Post-Encroachment Times for Bicycle-Vehicle Interactions Observed in the Field, a Driving Simulator, and in Traffic Simulation Models, Portland State University, Portland, OR, USA, 2016.

[17] L. Yang, J. H. Yang, E. Feron, and V. Kulkarni, “Development of a performance-based approach for a rear-end collision warning and avoidance system for automobiles," in Proceedings of the IEEE IV2003 Intelligent Vehicles Symposium Proceedings (Cat No 03TH8683), pp. 316-321, IEEE, Columbus, OH, USA, 2003.

[18] Y. Wu, M. Abdel-Aty, J. Park, and J. Zhu, "Effects of crash warning systems on rear-end crash avoidance behavior under fog conditions," Transportation Research Part C: Emerging Technologies, vol. 95, pp. 481-492, 2018.

[19] Y. Wu, M. Abdel-Aty, O. Zheng, Q. Cai, and L. Yue, "Developing a crash warning system for the bike lane area at intersections with connected vehicle technology," Transportation Research Record: Journal of the Transportation Research Board, vol. 2673, no. 4, pp. 47-58, 2019.

[20] C. Oh, S. Park, and S. G. Ritchie, "A method for identifying rear-end collision risks using inductive loop detectors," Accident Analysis \& Prevention, vol. 38, no. 2, pp. 295-301, 2006.

[21] L. Yue, M. Abdel-Aty, Y. Wu, J. Yuan, and M. Morris, "Influence of pedestrian-to-vehicle technology on drivers' response and safety benefits considering pre-crash conditions," Transportation Research Part F: Traffic Psychology and Behaviour, vol. 73, pp. 50-65, 2020.

[22] R. Wu, X. Zheng, Y. Xu et al., "Modified driving safety field based on trajectory prediction model for pedestrian-vehicle collision," Sustainability, vol. 11, no. 22, p. 6254, 2019.

[23] M. Fallah Zavareh, A. R. Mamdoohi, and T. Nordfjærn, "The effects of indicating rear-end collision risk via variable message signs on traffic behaviour," Transportation Research Part F: Traffic Psychology and Behaviour, vol. 46, pp. 524-536, 2017.

[24] T. M. Ruff and T. P. Holden, "Preventing collisions involving surface mining equipment: a GPS-based approach," Journal of Safety Research, vol. 34, no. 2, pp. 175-181, 2003.

[25] J. Wu, H. Xu, Y. Zhang, and R. Sun, "An improved vehiclepedestrian near-crash identification method with a roadside LiDAR sensor," Journal of Safety Research, vol. 73, pp. 211224, 2020.

[26] P. Chen, W. Zeng, G. Yu, and Y. Wang, "Surrogate safety analysis of pedestrian-vehicle conflict at intersections using unmanned aerial vehicle videos," Journal of Advanced Transportation, vol. 2017, Article ID 5202150, 12 pages, 2017.

[27] J. Li, B. Dai, X. Li, X. Xu, and D. Liu, "A dynamic Bayesian network for vehicle maneuver prediction in highway driving scenarios: framework and verification," Electronics, vol. 8, no. 1, p. 40, 2019.

[28] H. Araki, K. Yamada, Y. Hiroshima, and T. Ito, "Development of rear-end collision avoidance system," in Proceedings of the Conference on Intelligent Vehicles, pp. 224-229, IEEE, Tokyo, Japan, 1996.

[29] L. García Cuenca, E. Puertas, J. Fernandez Andrés, and N. Aliane, "Autonomous driving in roundabout maneuvers using reinforcement learning with Q-learning," Electronics, vol. 8, no. 12, p. 1536, 2019.

[30] M. Jannat, S. M. Roldan, S. A. Balk, and K. Timpone, "Assessing potential safety benefits of advanced pedestrian technologies through a pedestrian technology test bed," Journal of Intelligent Transportation Systems, vol. 25, no. 2, pp. 1-18, 2020. 
[31] R. Layton and K. Dixon, Stopping Sight Distance, Kiewit Center for Infrastructure and Transportation, Oregon Department of Transportation, Clackamas, OR, USA, 2012.

[32] M. M. Minderhoud and P. H. L. Bovy, "Extended time-tocollision measures for road traffic safety assessment," Accident Analysis \& Prevention, vol. 33, no. 1, pp. 89-97, 2001.

[33] Y. Zhang, E. K. Antonsson, and K. Grote, "A new threat assessment measure for collision avoidance systems," in Proceedings of the 2006 IEEE Intelligent Transportation Systems Conference, pp. 968-975, IEEE, Toronto, Canada, 2006.

[34] H. Kim, X. Wu, J. L. Gabbard, and N. F. Polys, "Exploring head-up augmented reality interfaces for crash warning systems," in Proceedings of the 5th International Conference on Automotive User Interfaces and Interactive Vehicular Applications, pp. 224-227, Eindhoven, Netherlands, 2013.

[35] J. A. Hanley and B. J. Mcneil, "The meaning and use of the area under a receiver operating characteristic (ROC) curve," $R a$ diology, vol. 143, no. 1, pp. 29-36, 1982.

[36] R. A. Shaikh and V. Thayananthan, "Risk-based decision methods for vehicular networks," Electronics, vol. 8, no. 6, p. 627, 2019.

[37] C. Wang, L. Ma, R. Li, T. S. Durrani, and H. Zhang, "Exploring trajectory prediction through machine learning methods," IEEE Access, vol. 7, pp. 101441-101452, 2019.

[38] Z. Cheng, Y. Li, and B. Wu, "Early warning method and model of inland ship collision risk based on coordinated collisionavoidance actions," Journal of Advanced Transportation, vol. 2020, Article ID 5271794, 14 pages, 2020.

[39] J. Raj, K. Raghuwaiya, and J. Vanualailai, "Collision avoidance of $3 \mathrm{D}$ rectangular planes by multiple cooperating autonomous agents," Journal of Advanced Transportation, vol. 2020, Article ID 4723687, 13 pages, 2020.

[40] J. Li, H. Wang, W. Zhao, and Y. Xue, "Ship's trajectory planning based on improved multiobjective algorithm for collision avoidance," Journal of Advanced Transportation, vol. 2019, Article ID 4068783, 12 pages, 2019. 